\title{
In situ effect of CPP-ACP chewing gum upon erosive enamel loss
}

\section{Abstract}

Catarina Ribeiro Barros de ALENCAR ${ }^{1}$

Gabriela Cristina de OLIVEIRA ${ }^{2}$

Ana Carolina MAGALHÃES ${ }^{3}$

Marília Afonso Rabelo BUZALAF ${ }^{3}$

Maria Aparecida de Andrade

Moreira MACHADO²

Heitor Marques HONÓRIO²

Daniela RIOS 2
Submitted: August 24, 2016 Modification: October 31, 2016 Accepted: November 15, 2016

Corresponding address: Dr. Daniela Rios

Bauru Dental School - University of São Paulo Department of Pediatric Dentistry, Orthodontics and Public Health Al. Dr. Octávio Pinheiro Brisolla, 9-75 Bauru - SP - Brazil - 17012-101 - C.P. 73 Phone/Fax: +55 14 3235-8224 e-mail: danirios@usp.br
Casein phosphopeptide-amorphous calcium phosphate (CPP-ACP) is able to increase salivary calcium and phosphate levels at an acidic $\mathrm{pH}$. Previous studies demonstrated that a CPP-ACP chewing gum was able to enhance the re-hardening of erosion lesions, but could not diminish enamel hardness loss. Therefore, there is no consensus regarding the effectiveness of CPPACP on dental erosion. Objective: This in situ study investigated the ability of a CPP-ACP chewing gum in preventing erosive enamel loss. Material and Methods: During three experimental crossover phases (one phase per group) of seven days each, eight volunteers wore palatal devices with human enamel blocks. The groups were: GI - Sugar free chewing gum with CPP-ACP; GII - Conventional sugar free chewing gum; and GIII - No chewing gum (control). Erosive challenge was extraorally performed by immersion of the enamel blocks in cola drink ( $5 \mathrm{~min}, 4 \mathrm{x} /$ day). After each challenge, in groups CPP and No CPP, volunteers chewed one unit of the corresponding chewing gum for 30 minutes. Quantitative analysis of enamel loss was performed by profilometry $(\mu \mathrm{m})$. Data were analyzed by Repeated-Measures ANOVA and Tukey's test $(p<0.05)$. Results: The use of chewing gum (CPP and No $\mathrm{CPP}$ ) resulted in lower erosive enamel loss compared with the control group $(p<0.05)$. CPP-ACP chewing gum (CPP) did not improve the protection against erosive enamel loss compared with conventional chewing gum (No CPP) $(p>0.05)$. Conclusion: The CPP-ACP chewing gum was not able to enhance the anti-erosive effect of conventional chewing gum against enamel loss.

Keywords: Tooth erosion. Dental enamel. Tooth wear. Chewing gum. Casein phosphopeptide-amorphous calcium phosphate.

${ }^{1}$ Universidade Estadual da Paraíba, Departamento de Odontologia, Araruna, PB, Brasil 2Universidade de São Paulo, Faculdade de Odontologia de Bauru, Departamento de Odontopediatria, Ortodontia e Saúde Coletiva, Bauru, SP, Brasil

3Universidade de São Paulo, Faculdade de Odontologia de Bauru, Departamento de Ciências Biológicas, Bauru, SP, Brasil 


\section{Introduction}

Tooth wear is a progressive and multifactorial process in which erosion, attrition, and abrasion may synergistically act ${ }^{25}$. Although there is no consensus on the diagnostic criteria that differentiates these lesions it has been considered that erosion is the largest contributing factor to the tooth wear in childhood and adolescence ${ }^{4}$. The term erosive tooth wear refers to chemical-mechanical processes in which abrasive forces removes the softened layer attacked by nonbacterial acids, causing tooth hard substance loss $^{12}$. However, tooth hard tissue loss can also occur without significant involvement of abrasion, in cases of prolonged repeated erosive challenges ${ }^{12}$

Adequate preventive measures will often decrease the erosion progression and reduce the need for immediate restorations ${ }^{11}$. The control of dental erosion requires a combination of strategies that includes the reduction on the frequency of erosive challenges and the enhancement of salivary defenses ${ }^{12}$. The latter can be achieved, among other ways, by the use of chewing gum ${ }^{22}$. In addition, therapies including fluoride applications, in high-concentration, acidic formulations and polyvalent sources like as stannous fluoride have been described to yield positive results in erosive treatment ${ }^{8}$.

Based on CPP-ACP promising results for subsurface carious lesions treatment ${ }^{9,14}$, some studies have been conducted to test this agent against dental erosion when added to pastes or mousses ${ }^{16,19,30}$, sports drinks ${ }^{18}$ or soft drinks ${ }^{6,13}$, and contained in chewing gum $^{1,10,17}$. However, there is no consensus regarding the effectiveness of CPP-ACP on dental erosion and its mechanism of action in relation to erosion is not fully understood ${ }^{30}$.

At an acidic $\mathrm{pH}$, amorphous calcium phosphate (ACP) will separate from casein phosphopeptide (CPP), thereby increasing salivary calcium and phosphate levels. The localized increase of calcium and phosphate degree of saturation will promote carious lesion remineralization by the diffusion of the higher concentration gradient to the lowest ${ }^{21}$. Confirming this effect, in previous studies of erosion, the in situ use of chewing gum with CPP-ACP was able to enhance the rehardening of erosion lesions ${ }^{1,17}$. Additionally, the increase of salivary calcium and phosphate levels by CPP-ACP at acidic $\mathrm{pH}$ might also inhibit demineralization ${ }^{20}$. Recently, an in situ study showed that the use of chewing gum immediately before a short erosive demineralization was able to diminish enamel hardness loss, however, the presence of CPP-ACP in the chewing gum could not enhance this protective effect ${ }^{10}$. It is important to point out that in situ models simulating initial erosion can give the first insights regarding preventive measures, but the interplay of erosive challenges and the protective mechanisms of the oral cavity along time are important to confirm the treatment effect. This study was designed to evaluate the effect of CPP-ACP chewing gum, considering the role process of erosion, trying to clarify the impact of remineralization and demineralization on the final degree of enamel loss. Therefore, the aim was to investigate the ability of CPP-ACP chewing gum in preventing enamel loss by erosive challenges. The null hypothesis tested was that there would be no difference between the CPP-ACP chewing gum and a conventional sugar free chewing gum in reducing erosive enamel loss.

\section{Material and Methods}

\section{Ethical standards}

Ethical approval for the study was granted by the local Institutional Ethics Committee (protocol no. 169). This study was conducted in full accordance with the Declaration of Helsinki. Eight healthy adult subjects (six female and two males) with an average age of 27.2 years (range $23-38$ years) took part of this study after giving informed consent form. Volunteers fulfilled the inclusion criteria: residing in the same fluoridated area $(0.70 \mathrm{mg} \mathrm{F} / \mathrm{I})$, physiological stimulated salivary flow rate $(>1 \mathrm{ml} / \mathrm{min}$ ), and adequate oral health (with no caries or erosion lesions). They also did not violate the exclusion criteria: systemic illness, pregnancy or breastfeeding, under orthodontic intervention, and use of fluoride compounds in the last two months.

\section{Experimental design}

The experimental design is illustrated in Figure 1. The study was conducted with a single-blind and randomized protocol of three-way crossover phases of seven days with an interval of one week between them. The factor under evaluation was treatment at three levels (GI - sugar free chewing gum with 18.8 mg of CPP-ACP; GII - conventional sugar free chewing gum without CPP-ACP; and GIII - negative control group, without chewing gum). Main components 
of the chewing gums are described in Figure 2. Eight volunteers wore acrylic palatal devices, each containing two human enamel blocks. Four daily erosive cycles (Coca Cola ${ }^{\circledR}$ Ribeirão Preto, Brazil, $\mathrm{pH} 2.4,0.32 \mathrm{ppm} \mathrm{F}$ ) were performed, after which the palatal devices were washed in running water. Thereafter, volunteers replaced the appliance into the mouth and chewed one unit of the chewing gum (Trident Total ${ }^{\circledR}$ or Trident Fresh ${ }^{\circledR}$ - Kraft Foods/Cadbury Adams, Bauru, SP, Brazil for groups CPP and No CPP, respectively) for 30 minutes. Quantitative analysis of enamel loss was performed by profilometry.

\section{Enamel samples preparation}

Enamel blocks $(4 \times 4 \times 3 \mathrm{~mm})$ were obtained from unerupted human third molars recently extracted and kept in $0.1 \%$ thymol solution at $\mathrm{pH} 7.0$. The blocks were cut using a ISOMET low speed saw cutting machine (Buehler Ltd., Lake Bluff, IL, USA) with two diamond disks (Extec Corp., Enfield, CT, USA), which were separated by a $4 \mathrm{~mm}$ thickness spacer. The surfaces of the blocks were ground flat with watercooled silicon carbide discs (320,600, and 1200 grade papers; Buehler, Lake Bluff, IL, USA) and polished with felt paper wet by diamond spray ( $1 \mu \mathrm{m}$ polishing particle size; Buehler, Ltd., Lake Bluff, IL, USA). Blocks were cleaned using an ultrasonic device for 10 minutes and checked regarding the presence of white spots and cracks using a microscope (x40).

The surface hardness ( $\mathrm{SH}$ ) was determined using the average values of five indentations performed at distances of $100 \mu \mathrm{m}$ from each other (Knoop diamond, $25 \mathrm{~g}, 10 \mathrm{~s}$, Hardness tester from Buehler, US). Seventy-two enamel blocks with mean $\mathrm{SH}$ of
343.9 ( $\pm 15.5 \mathrm{KHN}$ ) were selected and sterilized by exposure to ethylene oxide gas.

\section{First profile evaluation}

Enamel blocks were marked with a scalpel blade No. 11 (Embramac, Itapira, SP, Brazil) for standardization of two control areas with width of $1.0 \mathrm{~mm}$ (edges) and one test area with $2.0 \mathrm{~mm}$ (center). The baseline profile of enamel blocks was evaluated by a contact profilometer (Marh, MarSurf GD 25, Göttingen, Germany) using a contour software (MarSurf XCR 20). Blocks were fixed in a device to standardize their position and to allow the record of the location of each profile. In each block, five readings were made at predetermined distances of 2.25, 2.0, 1.75, 1.5, and $1.25 \mu \mathrm{m}$. The graphics (profile) of each read were individually saved. Then, control areas located on the two-thirds in the edges of the blocks were protected with cosmetic nail varnish (Maybelline Colorama, Cosbra Cosmetics Ltda, São Paulo, SP, Brazil) to maintain their integrity during the in situ phase.

\section{Sample size calculation}

Sample size calculation was based on a pilot in situ study with three volunteers. A sample size of eight volunteers was estimated based on a a-error of $5 \%, \beta$-error of $20 \%, 0.9 \mu \mathrm{m}$ as estimated standard deviation, and $1.51 \mu \mathrm{m}$ as minimum detectable means difference.

\section{Volunteers and in situ phase}

The intraoral palatal devices were made of the upper arch for each volunteer with acrylic resin on the plaster model. Each palatal device had two vertical

Figure 2- Main components of the chewing gums under study, according to manufactures information

\begin{tabular}{|c|c|}
\hline Trident Total $^{\circledR}$ (CPP-ACP)* & Trident Fresh ${ }^{\circledR}$ (without CPP-ACP)* \\
\hline Gum base & Gum base \\
\hline $\begin{array}{l}\text { Sweeteners: Sorbitol, manitol, maltitol, aspartame, acesulfame-k } \\
\text { and sucralose }\end{array}$ & $\begin{array}{c}\text { Sweeteners: Sorbitol, manitol, maltitol, xylitol, aspartame, } \\
\text { acesulfame-k and sucralose }\end{array}$ \\
\hline Flavoring agents & Flavoring agents \\
\hline Humectant: Glycerin, triacetin & Humectant: Glycerin \\
\hline Emulsifier: Soylecithin & Emulsifier: Soylecithin \\
\hline Dyes: Titanium dioxide, brilliant blue fcf & $\begin{array}{l}\text { Dyes: Titanium dioxide, patent blue } \mathrm{V} \text {, anthocyanins, brilliant blue } \\
\text { fcf }\end{array}$ \\
\hline \multicolumn{2}{|l|}{ Thickener: Gum Arabic } \\
\hline \multicolumn{2}{|l|}{ Glazing Agent: Carnauba wax } \\
\hline $\begin{array}{l}\text { Casein phosphopeptide - amorphous calcium phosphate } \\
\text { (Recaldent }{ }^{\circledR} 18.8 \text { mg CPP-ACP/gum) }\end{array}$ & \\
\hline
\end{tabular}

*Information given by Trident, Cadbury Adams (Indústria e Comércio, Bauru, São Paulo, Brazil). 
rows, one on the right and the other on the left side, with one cavity $(6 \times 6 \times 3 \mathrm{~mm})$ in each side, for enamel blocks fixation. Samples were fixed with wax and were carefully adapted to the level of the resin surface of the acrylic device, in order to avoid dental plaque accumulation.

Seven days prior to and during the experiment period, the volunteers brushed their teeth with standardized fluoride toothpaste (Total 12, 1.100 ppm F, Colgate, Brazil) three times a day. The volunteers were instructed not to use any other fluoride product.

Intraoral palatal devices were installed at the day before the start of the experiment, in the evening after the last oral hygiene to allow the formation of acquired enamel pellicle. During the following seven days, erosive challenges were extraorally performed four times a day ( $8 \mathrm{~h}, 12 \mathrm{~h}, 16 \mathrm{~h}$, and $20 \mathrm{~h}$ ). In each challenge, the palatal device was immersed in a cup containing $150 \mathrm{~mL}$ of a freshly opened bottle of a cola soft drink (Coca Cola ${ }^{\circledR}$ Ribeirão Preto, Brazil, pH 2.4, $0.32 \mathrm{ppm} F$ ) for 5 minutes.

After the erosive challenge, intraoral devices were washed in tap water and reinserted into the oral cavity. Then, for GI (Sugar free chewing gum with CPP-ACP) and GII (Conventional sugar free chewing gum), the volunteers chewed one unit of the respective gum for
30 minutes. After this time, the chewing gum was discarded, and the appliance was worn for at least 2 hours before its removal for another immersion in erosive beverage or for eating.

Volunteers were instructed to use the intraoral palatal device continuously (20 h/day) except during meals (four meals daily of 1 hour each). In the periods of range of use, the appliances were kept in a plastic box, wrapped in gauze moistened in water supply (Bauru, São Paulo, Brazil - 0.7 ppm F).

\section{Final prolife evaluation and enamel loss measurement}

After the in situ phase, enamel blocks were removed from intraoral palatal devices. The cosmetic nail varnish was mechanically removed by using the tip of a scalpel blade positioned in the angle between the base and external wall of the block surface. Enamel blocks were repositioned on the table of the profilometer according to its baseline position and five readings were performed as previously described. Baseline and final graphs (profile) of each one of the five readings were superimposed using MarSurf XCR 20 software (Figure 1 ). The average points were selected for measuring the distance between the graphs in height, in order to define the enamel loss

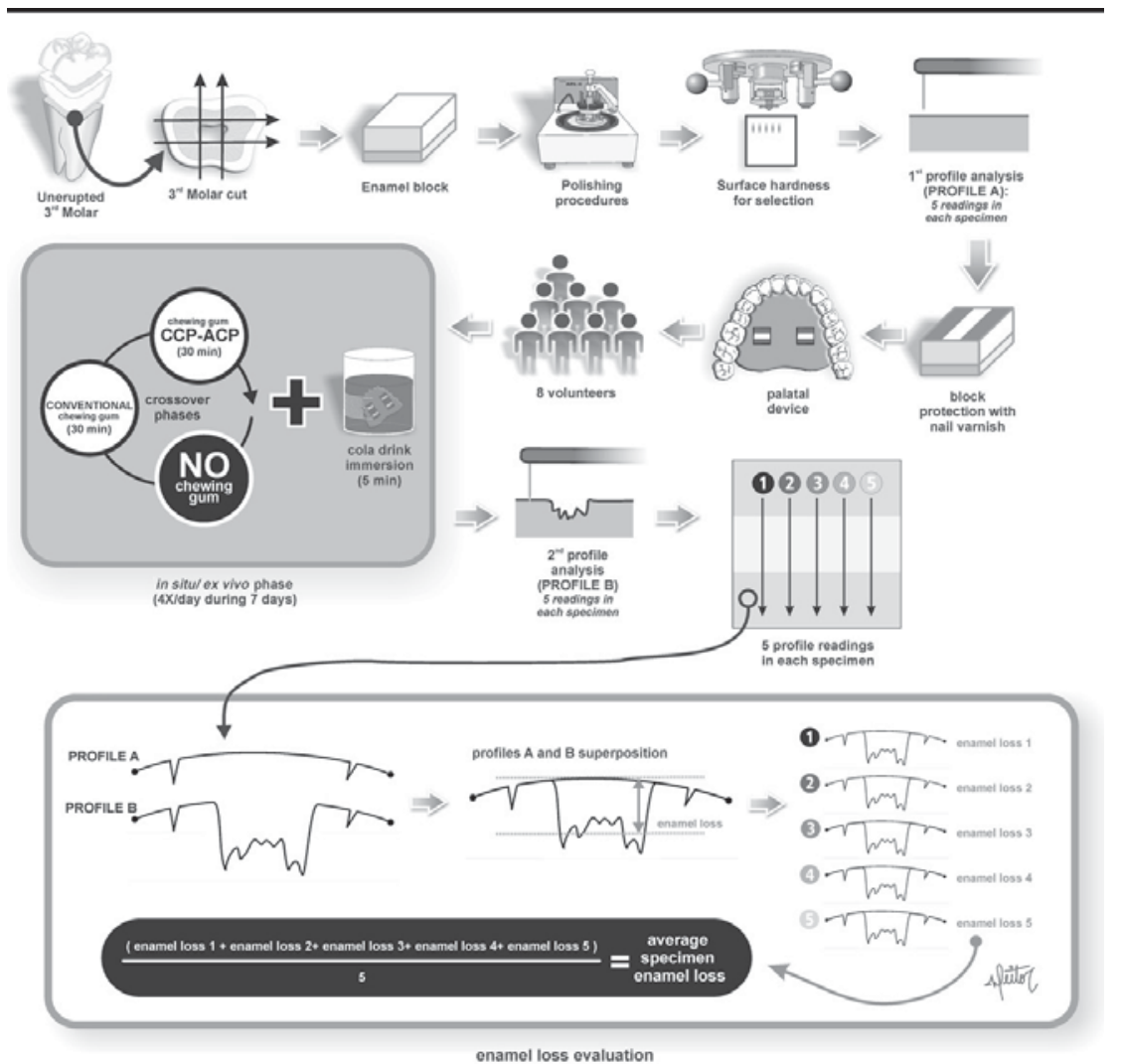

Figure 1- Flowchart of the experimental design 
Table 1- Means and standard deviation values of erosive enamel loss $(\mu \mathrm{m})$ for experimental groups

\begin{tabular}{cc}
\hline Experimental Groups & Mean \pm SD \\
\hline GI (CPP-ACP) & $5.2 \pm 2.8^{\mathrm{a}}$ \\
GII (without CPP-ACP) & $3.8 \pm 1.5^{\mathrm{a}}$ \\
\hline GIII (no chewing gum) & $6.8 \pm 3.5^{\mathrm{b}}$ \\
\hline
\end{tabular}

Groups whose means are followed by distinct letters differ significantly. (Repeated Measures ANOVA/Tukey's Test, $p<0.05$ ).

in micrometers.

\section{Statistical analysis}

Statistical analysis was performed with SigmaPlot version 12.3 (2011 Systat Software, Germany). The assumption of equality of variances were satisfied and Shapiro-Wilk test checked the normal distribution of the data $(p>0.05)$. Repeated- Measures ANOVA followed by Tukey's test were applied. The significant limit was set at $5 \%$.

\section{Results}

All eight volunteers completed the in situ protocol and no side effects were reported. Table 1 shows that the chewing gum use (GI and GII) resulted in a significantly lower erosive enamel loss compared with the control group (GIII - without gum). Chewing gum containing CPP-ACP (GI) did not improve the protection against erosive enamel loss in comparison with the conventional chewing gum (GII) ( $p>0.05)$.

\section{Discussion}

The ideal prevention and control of erosive tooth wear involves the removal of causal factors. However, this approach is difficult and not always practiced. For this reason, different therapies have been proposed and studied $2,8,15$.

One of these therapies is the use of CPP-ACP. Promising results were obtained from studies that evaluated the CPP-ACP remineralizing capacity on eroded lesions ${ }^{1,16,17,19}$. On the other hand, other studies have found some limitation in the effect of CPP-ACP when the product was applied before the initial acid attack ${ }^{10}$ or in erosive cycling protocols, in which fluoride was used as the positive control ${ }^{28}$. The knowledge of the effect of chewing gum containing CPP-ACP on erosive lesions is still limited in the literature.
The results of the present study showed that the use of chewing gum diminished the enamel loss from erosive challenges when compared with no chewing gum. This is in accordance with previous findings reporting a reduction in erosive tooth wear with the use of sugar-free chewing gum for 30 minutes after erosive challenges ${ }^{22,23}$. The anti-erosive potential of different agents has been supported by the repair of eroded lesions as a result of mineral precipitation ${ }^{2}$. Based on this, it was hypothesized that the protective effect of the chewing gum was due to mechanical and gustatory salivary stimulation that elevate calcium concentrations in saliva, promoting mineral deposition on the eroded lesion, thereby reducing enamel loss in subsequent acid attack. However, the magnitude of the protection by mineral precipitation can be questioned when considering overall erosion prevention. The histological features of eroded enamel indicate that part of the tissue is etched away during the erosive attack resulting in limited areas for remineralization to take place 25 .

There are two studies that showed an improvement on rehardening (remineralization) of eroded enamel after the use of chewing gum containing CPP-ACP in comparison to the salivary stimulus by regular gum ${ }^{1,17}$. The effect of casein on enamel remineralization has been associated with a subsequent increase in tissue resistance and reduced solubility of tooth structures to acid attack ${ }^{9}$. However, when considering cycles of demineralization and remineralization, as applied in the present study, the previously found rehardening effect of CPP-ACP on eroded enamel did not reflect in higher enamel resistance against erosive enamel loss when compared with the effect promoted by salivary stimulus using a regular gum. Results did not show differences in the erosion-preventive effect between regular and CPP-ACP containing gums. Therefore, there is no evidence that higher mineral precipitation, as promoted by CPP-ACP gums, will provide a less susceptible enamel surface against subsequent erosive challenges.

As suggested by Eisenburger, et al. ${ }^{5}$ (2001), in vitro remineralization of the eroded surface after 1 , 2 , and 4 hours in artificial saliva is extremely fragile and susceptible to removal by mechanical forces. The remineralization process would involve the deposition of minerals in zones of enamel porosity rather than the promotion of regeneration of hydroxyapatite crystals in these short periods of remineralization. 
However, as erosive lesions are vulnerable to other damage immediately after its formation, the goal of remineralization of erosion lesions would be restore mechanical strength as much as possible in order to avoid subsequent losses; though evidence has shown that it is far from being achieved ${ }^{26}$.

When considering erosive enamel loss the most important effect of preventive treatments seems to be surface protection rather than mineral precipitation ${ }^{8}$. The mechanism of enamel protection of CPP-ACP is based on the increase of the degree of saturation of calcium and phosphate when $\mathrm{pH}$ drops by erosive challenge, inhibiting demineralization ${ }^{29}$. In addition, it is hypothesized that CPP-ACP ability of forming micelles on the tooth surface ${ }^{3}$ could result in a semipermeable barrier ${ }^{24}$, which might restrict the reaction of $\mathrm{H}^{+}$ion with the tooth surface and also inhibit the loss of $\mathrm{Ca}^{+2}$ ions. However, Jordão, et al. ${ }^{10}$ (2016) found that the use of chewing gum immediately before an erosive demineralization can diminish enamel hardness loss and the presence of CPP-ACP in the chewing gum cannot enhance this protective effect. These results were similar to the present study, reinforcing the theses that CPP-ACP is not able to protect enamel against erosion. In contrast, de Oliveira, et al. ${ }^{15}$ (2017), using similar in situ protocol, showed that the same commercial CPP-ACP chewing gum resulted in lesser enamel loss compared with regular chewing gum when enamel blocks were subjected to erosion. On the other hand, when erosion was associated to abrasion, the gums showed statistically similar behavior. Therefore, in a more realistic condition, in which erosive enamel wear is enhanced by oral mechanical forces, CPP-ACP shows limited effect. Since in the present study there was no apparatus on the palatal appliance to hinder the forces of the tongue $e^{7,27}$, it is speculated that erosion was associated to tongue abrasion and the addition of CPP-ACP was not able to enhance the protection promoted by a conventional chewing gum use, similarly to the results of Oliveira, et al. ${ }^{15}$ (2017).

Based on the results of the present study, the inclusion of CPP-ACP in a chewing gum was not able to enhance the protective effect of the use of a conventional chewing gum against erosive enamel loss. The null hypothesis was accepted. Therefore, to date, there is no evidence that chewing gum containing CPP-ACP should be used to prevent the development or progression of erosive enamel loss.

\section{Acknowledgements}

The authors would like to gratefully acknowledge all the volunteers who participated in this study and FAPESP - São Paulo Research Foundation for the financial support (Proc. no. 2011/16326-7). The funders had no role in study design, data collection and analysis, decision to publish, or preparation of the manuscript.

\section{References}

1- Alencar CR, Magalhães AC, Machado MA, Oliveira TM, Honório HM, Rios D. In situ effect of a commercial CPP-ACP chewing gum on the human enamel initial erosion. J Dent. 2014;42(11):1502-7.

2- Buzalaf MA, Magalhães AC, Wiegand A. Alternatives to fluoride in the prevention and treatment of dental erosion. Monogr Oral Sci. 2014;25:244-52.

3- Devold TG, Rykke M, Isabey D, Sørensen ES, Christensen B, Langsrud $\mathrm{T}$, et al. In vitro studies of adsorption of milk proteins onto tooth enamel. Int Dairy J. 2006;16:1013-7.

4- Dugmore CR, Rock WP. The prevalence of tooth erosion in 12-yearold children. Br Dent J. 2004;196(5):279-82.

5- Eisenburger M, Addy M, Hughes JA, Shellis RP. Effect of time on the remineralization of enamel by synthetic saliva after citric acid erosion. Caries Res. 2001;35(3):211-5.

6- Ferrazzano GF, Coda M, Cantile T, Sangianantoni G, Ingenito A. SEM investigation on casein phosphopeptides capability in contrasting cola drinks enamel erosion: an in vitro preliminary study. Eur J Paediatr Dent. 2012;13(4):285-8.

7- Gregg T, Mace S, West NX, Addy M. A study in vitro of the abrasive effect of the tongue on enamel and dentine softened by acid erosion. Caries Res. 2004;38(6):557-60. 42

8- Huysmans MC, Young A, Ganss C. The role of fluoride in erosion therapy. Monogr Oral Sci. 2014;25:230-43.

9- Iijima Y, Cai F, Shen P, Walker G, Reynolds C, Reynolds EC. Acid resistance of enamel subsurface lesions remineralized by a sugar-free chewing gum containing casein phosphopeptide-amorphous calcium phosphate. Caries Res. 2004;38(6):551-6.

10- Jordão MC, Alencar CR, Mesquita IM, Buzalaf MA, Magalhães AC, Machado MA, et al. In situ Effect of chewing gum with and without CPPACP on enamel surface hardness subsequent to ex vivo acid challenge. Caries Res. 2016;50(3):325-30.

11- Lussi A, Hellwig E. Risk assessment and causal preventive measures. Monogr Oral Sci. 2014;25:220-9.

12- Lussi A, Carvalho TS. Erosive tooth wear: a multifactorial condition of growing concern and increasing knowledge. Monogr Oral Sci. 2014;25:1-15.

13- Manton DJ, Cai F, Yuan Y, Walker GD, Cochrane NJ, Reynolds C, et al. Effect of casein phosphopeptide-amorphous calcium phosphate added to acidic beverages on enamel erosion in vitro. Aust Dent $\mathrm{J}$. 2010;55(3):275-9.

14- Morgan MV, Adams GG, Bailey DL, Tsao CE, Fischman SL, Reynolds EC. The anticariogenic effect of sugar-free gum containing CPP-ACP nanocomplexes on approximal caries determined using digital bitewing radiography. Caries Res. 2008;42(3):171-84.

15- Oliveira AF, Oliveira Diniz LV, Forte FD, Sampaio FC, CcahuanaVásquez RA, Tochukwu Amaechi B. In situ effect of a CPP-ACP chewing gum on enamel erosion associated or not with abrasion. Clin Oral Investig. 2017;21(1):339-46

16- Poggio C, Lombardini M, Vigorelli P, Ceci M. Analysis of dentin/ enamel remineralization by a CPP-ACP paste: AFM and SEM study. Scanning. 2013;35(6):366-74. 
17- Prestes L, Souza BM, Comar LP, Salomão PA, Rios D, Magalhães AC. In situ effect of chewing gum containing CPP-ACP on the mineral precipitation of eroded bovine enamel-a surface hardness analysis. J Dent. 2013;41(8):747-51.

18- Ramalingam L, Messer LB, Reynolds EC. Adding casein phosphopeptide-amorphous calcium phosphate to sports drinks to eliminate in vitro erosion. Pediatr Dent. 2005;27(1):61-7.

19- Ranjitkar S, Kaidonis JA, Richards LC, Townsend GC. The effect of CPP-ACP on enamel wear under severe erosive conditions. Arch Oral Biol. 2009;54(6):527-32.

20- Reynolds EC, Cai F, Shen P, Walker GD. Retention in plaque and remineralization of enamel lesions by various forms of calcium in a mouthrinse or sugar-free chewing gum. J Dent Res. 2003;82(3):20611.

21- Reynolds EC. Casein phosphopeptide - Amorphous calcium phosphate: the scientific evidence. Adv Dent Res. 2009;21(1):25-9. 22- Rios D, Honório HM, Magalhães AC, Delbem AC, Machado MA, Silva SM, et al. Effect of salivary stimulation on erosion of human and bovine enamel subjected or not to subsequent abrasion: an in situ/ex vivo study. Caries Res. 2006;40(3):218-23.
23- Rios D, Honório HM, Magalhães AC, Silva SM, Delbem AC, Machado $M A$, et al. Scanning electron microscopic study of the in situ effect of salivary stimulation on erosion and abrasion in human and bovine enamel. Braz Oral Res. 2008;22(2):132-8.

24- Rykke M, Smistad G, Rölla G, Karsen J. Micelle like structures in human saliva. Colloids Surf B Biointerfaces. 1995;4(1):33-44.

25- Shellis RP, Addy M. The interactions between attrition, abrasion and erosion in tooth wear. Monogr Oral Sci. 2014;25:32-45

26- Shellis RP, Featherstone JD, Lussi A. Understanding the chemistry of dental erosion. Monogr Oral Sci. 2014;25:163-79.

27- Vieira A, Overweg E, Ruben JL, Huysmans MC. Toothbrush abrasion, simulated tongue friction and attrition of eroded bovine enamel in vitro. J Dent. 2006;34(5):336-42.

28- Wegehaupt FJ, Tauböck TT, Stillhard A, Schmidlin PR, Attin T. Influence of extra- and intra-oral application of CPP-ACP and fluoride on re-hardening of eroded enamel. Acta Odontol Scand. 2012;70(3):17783.

29- White AJ, Gracia LH, Barbour ME. Inhibition of dental erosion by casein and casein-derived proteins. Caries Res. 2011;45(1):13-20. 30- Wiegand A, Attin T. Randomised in situ trial on the effect of milk and CPP-ACP on dental erosion. J Dent. 2014;42(9):1210-5. 